\title{
Modelling Tunnel Thrusters for Autonomous Underwater Vehicles
}

\author{
Alistair Palmer*, Grant E. Hearn* \\ Peter Stevenson**
*Fluid Structure Interactions Research Group, School of Engineering Sciences, University of Southampton, SO171BJ,UK (e-mail:ap1601@soton.ac.uk,g.e.hearn@soton.ac.uk). ** Underwater Systems Laboratory, National Oceanography Centre, Southampton, SO14 3ZH, UK (e-mail:pst@noc.soton.ac.uk).

\begin{abstract}
With 900 Autonomous Underwater Vehicles (AUVs) required over the next decade (Newman et al., 2007) existing survey-style AUVs need improved utilization factors. Additional control devices to extend operational capability need consideration together with the interchange between AUV control approaches. This paper considers supplementary through-body tunnel thruster control during the transition from survey operation to low-speed manoeuvring. Modified manoeuvring equations permit investigation of energy level demands as a positively buoyant AUV is slowed down from cruising speed to maintaining a stationary position. A suitable model of the selected thruster device is proposed following a literature review of tunnel thruster performance.
\end{abstract}

Keywords: Autonomous Vehicles, Marine Systems, Propulsion Control, Manoeuvrability, Mathematical Models, Simulation.

\section{INTRODUCTION}

Survey-style Autonomous Underwater Vehicles (AUVs) commonly use a stern mounted propeller and control surfaces to provide depth and heading control at survey speed. The hydrostatic balance on a positively buoyant vehicle is controlled by operating at a small negative pitch angle, maintained by the control surfaces, to generate a downwards force hydrodynamically. The magnitude of the force generated is dependent upon the pitch angle and the flow speed.

To retain the existing survey efficiency and add low speed manoeuvring capabilities requires additional control devices and new control algorithms to augment the stern propeller and aerofoil based control surfaces used at survey speeds. Following an internal review of control devices, including internal actuation systems and externally mounted thruster arrangements, through-body tunnel thrusters were selected for further investigation because of their relative simplicity, responsiveness, and low drag penalty on survey operations. To maintain control over the entire speed range it is important to understand the operation and performance of the thruster in different operational conditions.

Tunnel thrusters use relatively large amounts of energy and the required energy varies with the operational conditions. Hence the limited energy supply onboard the AUV constrains the use of tunnel thrusters to low speed manoeuvring operations and the control of the hydrostatic balance for a positively buoyant AUV during the transition between survey operation and low speed manoeuvring. The latter application introduces a further problem, namely, how to interchange between control methods when going through the transition period.

\section{OPERATION AND PERFORMANCE OF TUNNEL THRUSTERS}

The steady state performance of the thruster is now addressed at zero speed of AUV advance, for an AUV undergoing forward motion, and for an AUV undergoing arbitrary motion.

\subsection{Performance at Zero Speed of Advance}

The performance of a tunnel thruster at zero speed of advance is analogous to the static conditions used in thruster characterisation experiments and thus the thrust generated can be expected to be proportional to the square of the thruster rotational speed (Carlton, 2007).

\subsection{Performance on a Vehicle Undergoing Forward Motion}

The performance of a tunnel thruster on a vehicle undergoing forward motion is more complicated due to the interaction of the ambient flow around the vehicle with the jet emitted from the thruster exit. Example experimental results for operation in this condition include Chislett \& Björheden (1966), which show a large decrease in the force experienced by the vehicle compared to the equivalent static thrust. Figure 1 shows this decrease for a submersible, giving the effective force as a fraction of the equivalent static thrust, $K_{F}$, against the speed ratio of the vehicle speed, $u$, to the thruster exit jet speed, $u_{j}$.

The decrease in the effective force is not due to a change in the performance of the thruster unit itself, rather more, it is attributed to the complex flow conditions generated by the interaction of the thruster exit jet flow with the ambient flow around the vehicle (English, 1963). (The flow conditions at the inlet are not thought to have a significant impact on the effective force (Brix \& Bussemaker, 1973).) The interaction 
causes a low pressure region downstream of the jet exit which creates a suction force that opposes the thruster force.

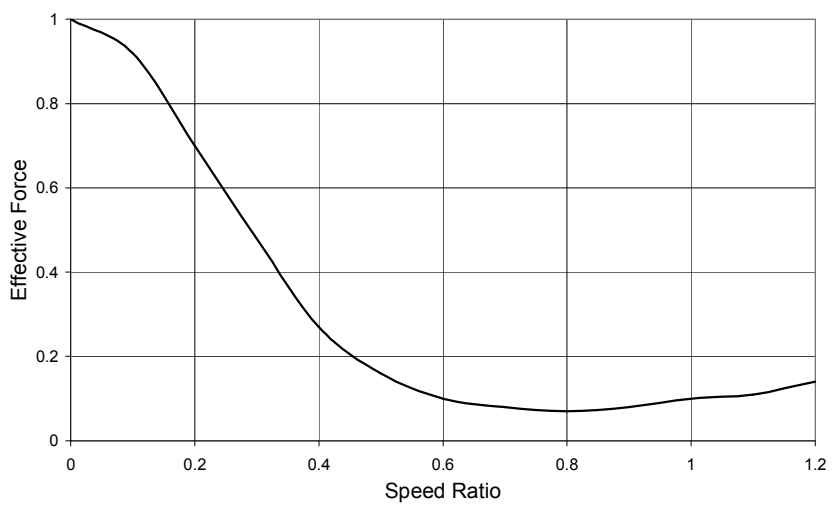

Figure 1 - Variation in effective force (vertical axis) with speed ratio (horizontal axis)

Figure 1 shows data presented in terms of the speed ratio, however some authors have presented data to show that the decrease in effective force is solely a function of the ambient flow speed and therefore independent of the jet speed (and hence thrust) (Brix \& Bussemaker, 1973; Karlikov \& Sholomovich, 1998). In addition to this, detailed experimental studies of jets emitting from flat plates into ambient flows have shown that for small speed ratios the jet dominates the ambient flow and hence the low pressure region is created solely by the separation of the ambient flow boundary layer (Gopalan et al., 2004; Fric \& Roshko, 1994). That is, for low speed ratios the reduction in effective force is solely a function of the ambient flow speed and is independent of the jet exit speed. The limited range of use of a tunnel thruster on an energy limited AUV dictates that the speed ratio is likely to be low.

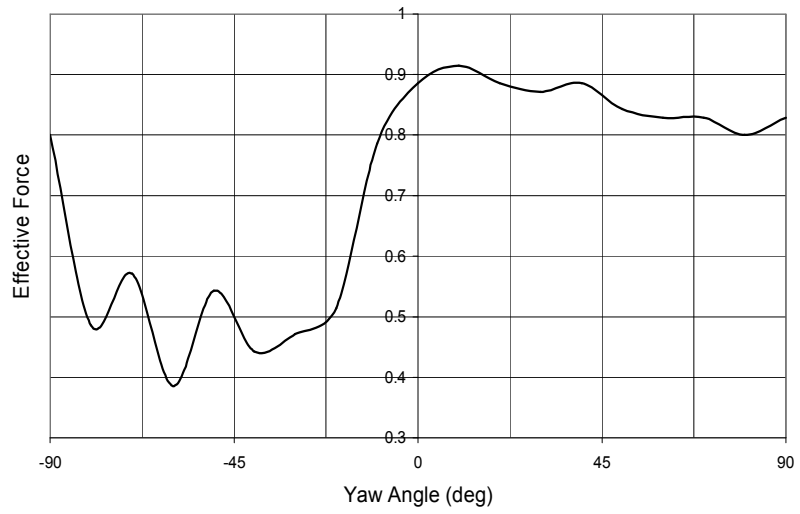

Figure 2 - Variation in effective force (vertical axis) with yaw angle in degrees (horizontal axis)

\subsection{Performance on a Vehicle Undergoing Arbitrary Motion}

The performance of a tunnel thruster on a vehicle undergoing arbitrary motion is more complicated due to the variation in the interaction of the ambient flow with the exit jet and the creation of an inflow component for the thruster. There is limited data for this mode of operation, however one data set for a surface vessel is shown in Figure 2, for a speed ratio of 0.2 (Symons \& Sadden, 1982). The performance in this mode can be separated into two different regions, firstly, thrusting against the ambient flow (positive yaw), and secondly, the more unlikely case of thrusting with the ambient flow (negative yaw), see Figure 3.

The former case, shown as positive yaw on Figures 2 and 3, implies that the ambient fluid flow is directed towards the inlet of the thruster. Consequently the vehicle nose tends to shield the exit region of the thruster and so removes the interaction between the ambient flow and the exit jet. In this case the decrease in effective force is small and occurs at a roughly steady rate. These characteristics suggest that the decrease in effective force is due to the variation in the performance of the thruster itself caused by the increasing flow component directed towards the thruster inlet. This conclusion is supported by existing data for the force generated by a tunnel thruster mounted in a torpedo-shaped AUV (Saunders \& Nahon, 2002). The cited data shows similar trends and magnitudes of thruster force variation to effective force variation shown in Figure 2.

The latter case implies that the ambient flow is directed towards the exiting jet causing complex interaction effects. All data sources for this type of flow condition agree that there is a large decrease in the effective force (Nienhuis, 1992). However the existing thruster force data (Saunders \& Nahon, 2002) does not exhibit the considerable decrease in effective force illustrated in Figure 2. This implies that the decrease is due to a complex interaction between the exit jet and the ambient flow, rather than the performance of the thruster.

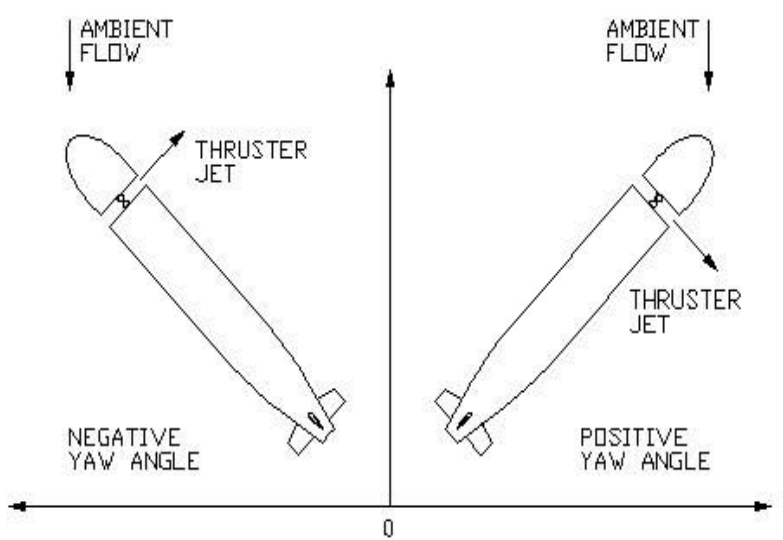

Figure 3 - Definition of flow conditions for vehicle undergoing arbitrary motion 


\section{CONTROL ARCHITECTURE}

In order for an AUV to operate effectively it is necessary for the control system to have knowledge of how the vehicle's thrusters perform. Therefore a model of the performance characteristics of the tunnel thrusters, as discussed in Section 2 , is required.

Recent developments in control systems for underwater vehicles have investigated control over the entire non-zero speed range incorporating the transition from the underactuated survey (high-speed) mode to the fully actuated low-speed mode (Breivik \& Fossen, 2006). These control architectures require knowledge of the forces and moments generated by a thruster with a given input command, as a function of vehicle motion and environmental conditions. Modelling of the thruster loads is considered next.

\section{MODELING APPROACHES FOR UNDERWATER THRUSTERS}

The steady state performance of propeller based thrusters has been studied extensively in the development of surface vessels (e.g. (van Lammeran et al., (1969)). However, the dynamic performance of a thruster can dominate the overall control system of an underwater vehicle at low speeds (Yoerger et al., 1990). Hence over the past two decades there has been a series of developments in the modelling of underwater thrusters focussing on their dynamic performance.

The first group of models were based on considering the thruster and tunnel as a control volume and applying momentum and energy theorems to derive relationships between the generated thrust, $F$, and torque, $Q$, and the flow through the thruster, $u_{p}$ (McLean, 1991; Cody, 1992). These relationships are combined with a model of the motor and blade element relations such that an example model can be summarised as (Healey et al., 1995):

$$
\begin{aligned}
& J_{m} \dot{n}+K_{n} n=Q_{m}-Q \\
& K_{1} \dot{u}_{p}+K_{2}\left(u_{p}-u\right)\left|u_{p}-u\right|=F \\
& F=F\left(n, u_{p}\right) \\
& Q=Q\left(n, u_{p}\right)
\end{aligned}
$$

Where $J_{m}$ is the motor inertia, $n$ is the rotational speed of the thruster, $Q_{m}$ is the motor control torque and $K_{n}, K_{l}$ and $K_{2}$ are constants. Recent developments of these models include more accurate representations of the lift and drag characteristics of the blade sections for use in the blade element relations for the functions $F$ and $Q$ (Bachmayer et al., 2000).

These improved models were found to work well with zero ambient flow, however their performance reduces in other conditions. This weakness led to the development of models which include a simplified model of the vehicle dynamics to estimate the flow into the thruster and the use of propeller characteristics, derived from open water charts (Blanke et al., 2000). Recent developments include more accurate representations of the propeller open water characteristics (Kim \& Chung, 2006).

\subsection{Previous Modelling Approaches for Tunnel Thrusters}

The original thruster models (McLean, 1991; Cody, 1992) were developed by considering and performing experiments using tunnel thrusters. However, the loss of performance when the ambient flow is no longer zero and the fact that effective force against the vehicle rather than actual thruster force is required meant that new models became desirable.

A literature survey for models of tunnel thruster performance on a moving underwater vehicle found only one model, which was developed using experimental data (Saunders \& Nahon, 2002). The authors tested the performance of the thruster in three different operating modes, namely, forward travel, low speed manoeuvring, and high speed turning. The basis model used was similar to that developed in (1) (Healey et al., 1995). It was found that the dynamic performance of the thruster was not significantly altered by the operating mode of the vehicle, however the steady state performance was affected. This led to the development of an augmented model:

$$
F=K_{1} \dot{u}_{p}+a+b n|n|,
$$

where $a$ and $b$ are determined from a look-up table of experimental results. An attempt to incorporate the vehicle forward speed into the basic model was found to be unsuccessful. The authors reported that the augmented model successfully captures the effects of forward speed and yaw angle on the performance of a tunnel thruster, however the model only considers the thruster forces and no account was made for the ambient flow effects.

Tunnel thrusters are similar in both their design and use to the secondary (commonly, bow) thrusters found on surface vessels. A literature survey for these types of models again yielded few results. A simple model, which calculates the force on the vessel depending on the vessel forward speed, but does not include yaw angle effects has the form:

$$
F=K_{3} n|n| \exp \left[-c u^{2}\right],
$$

where $K_{3}$ and $c$ are constants (Godhavn et al., 1998). This exponential form effectively models the variations in effective force when the force is decreasing, but deviates from the experimental data as the force recovers at higher advance speeds. Manoeuvring simulations performed at the Marine Research Institute Netherlands (MARIN) on vessels with secondary thrusters use a series of coefficients from look-up tables relating the performance under given conditions to the equivalent static performance as derived from a series of experiments on representative hull forms (Nienhuis, 1987).

\subsection{A Proposed AUV Tunnel Thruster Model}

The preceding sections and literature review have found no published and established modelling approach for tunnel thrusters, either on AUVs or surface vessels. It is believed that this is due to the complexity of the flow phenomena generated. A further contributing factor is the uncertainty over the effect of the operation of the tunnel thruster on the vehicle as a whole, for example, the change in performance 
of the vehicle due to the interaction of the exit jet flow with the vehicle downstream of the tunnel thruster.

To attempt to increase the understanding of the performance of a vehicle using tunnel thrusters and to gain insight into how to control an AUV it is important to be able to model the tunnel thruster as accurately as possible. To achieve this without conducting the complete range of experiments (which would facilitate the use of look-up tables), say for example, during the design phase, it would be useful to have a simple model that uses easily obtainable coefficients.

A key conclusion of Saunders \& Nahon (2002) was that the dynamic performance of the tunnel thruster was not significantly altered by the various ambient flow conditions tested. Therefore it is suggested that for the dynamic performance of the thruster any of the previously reported models can be employed, for example, (1). The steady state performance is greatly affected by the ambient flow conditions and hence it is suggested that this part of the model uses the following approach.

At zero (and very low) speed of advance the thruster can be assumed to operate as the static performance and thus the thrust can be assumed to be proportional to the square of the rotational speed, that is:

$$
F=K_{3} n|n| \text {. }
$$

On a vessel undergoing forward motion with a small yaw (or pitch) angle, $\psi\left(\psi_{L}<\psi<-\psi_{L}\right)$, the thruster performance is simply the static performance factored by an exponential reduction based on the forward speed. $\psi_{L}$ is a small negative yaw angle that represents a threshold over which the ambient flow component, perpendicular to the vehicle, is large enough to cause a decrease in the effective force such that the exponential model no longer accurately represents the variations observed. The exponential model uses the ambient flow component parallel to the vehicle, $u \cos (\psi)$, that is:

$$
F=K_{3} n|n| \exp \left[-c(u \cos (\psi))^{2}\right] .
$$

On a vessel undergoing arbitrary motion with the thruster thrusting against the ambient flow the operation can be assumed to vary as if undergoing an advance test, with a modified advance coefficient, $J$, in the form:

$$
J=\frac{u \sin (\psi)}{n D} .
$$

Here $D$ is the diameter of the tunnel thruster. This advance coefficient is based on the ambient flow component perpendicular to the vehicle, $u \sin (\psi)$, and can be used to determine the thrust and torque from a linear approximation of the open water characteristics using the effective force ratio, $K_{F}=(1-e J)$, that is:

$$
F=K_{3} n|n|(1-e J) .
$$

Here $e$ is an experimentally determined constant that can be obtained from a standard advance test and characterises the slope $d K_{F} / d J$.

On a vessel undergoing arbitrary motion with the thruster thrusting with the ambient flow $\left(\psi \leq \psi_{L}\right)$ the operation can be assumed to vary as the performance at a small yaw angle, $F_{\psi L}$, (to maintain continuity) factored by a sinusoidal function with a forward speed effect to model the decrease shown in Figure 2 for negative yaw angle. This gives:

$$
F=F_{\psi l}\left(1-g u\left|\sin \left[2\left(\psi-\psi_{L}\right)\right]\right|\right) .
$$

Here $g$ is a constant based on experimental results. It is important to emphasise that this condition is unlikely to be encountered during normal AUV operation. This is because thrusting with the ambient flow is unlikely to be necessary, that is, if the component of the ambient flow perpendicular to the vehicle is strong enough to significantly affect the performance of the thruster it is likely to be strong enough to manoeuvre the vehicle satisfactorily without the need for additional thrust.

These models of the performance can be combined to form a complete model, viz:

$$
\begin{aligned}
& \text { For } \psi \geq \psi_{L} \\
& \begin{array}{l}
F=K_{3} n|n|\left(1-e \frac{u|\sin (\psi)|}{n D}\right) \exp \left[-c(u \cos (\psi))^{2}\right] \\
\text { For } \psi \leq \psi_{L} \\
F=K_{3} n|n|\left(1-e \frac{u\left|\sin \left(\psi_{L}\right)\right|}{n D}\right) \exp \left[-c\left(u \cos \left(\psi_{L}\right)\right)^{2}\right] \\
\quad \cdot\left(1-g u\left|\sin \left[2\left(\psi-\psi_{L}\right)\right]\right|\right)
\end{array}
\end{aligned}
$$

The definition of the angle $\psi$ in (9) must account for the direction of the exit jet flow (see Figure 3).

\section{TUNNEL THRUSTER MODEL IMPLEMENTATION}

The model given in (9) has been implemented in a Matlab Simulink simulation of the Autosub AUV (Furlong, 2005) using the manoeuvring coefficients determined experimentally at Haslar (Kimber \& Marshfield, 1993) to test control strategies for the transition phase between survey operation and low speed operation. The numerical simulations performed model the vehicle undergoing a flightpath style deceleration, that is, decreasing speed at a slow and steady rate whilst maintaining depth. The depth control and hydrostatic balance control are undertaken using a pair of stern mounted control surfaces during survey operation. As the vehicle slows down the control surfaces lose their effectiveness and a new control method is required, chosen here to be a single, centrally mounted, tunnel thruster. The transition between these two control methods is undertaken using a function, $\sigma_{i}$, for the interchange between the two phases of operation (Breivik and Fossen, 2006). The proportion of the control, $\sigma_{i}$, at the $i$ th time step, given to each system is:

$$
\sigma_{i}(u)=1-0.5\left[\tanh \left(\frac{u_{i}-\bar{u}}{\Delta \sigma}\right)+1\right] .
$$

This model allows the designer to set the location of the transition zone, by selecting a mid-transition speed, $\bar{u}$, and set the 'steepness' of the transition, by altering $\Delta \sigma$. A low value of $\Delta \sigma$ gives a step jump at the mid-transition speed, whereas a high value gives a longer smooth transition. 
The aim of the simulations performed was to investigate how to select the transition control strategy. For a long range survey AUV a key factor to be minimised is the energy required for a certain manoeuvre. Therefore the energy used while slowing down is the chosen performance measure; it is a function of the mid-transition speed and the 'steepness' of the transition zone.

The chosen values for the model are shown in Table 1 and are based on matching experimental data. These coefficients are complex functions of the design and interaction of thruster and vehicle. Further details of the justification and verification of these choices cannot be presented here for space reasons. $\underline{\text { Table } 1 \text { - Model Coefficient Values }}$

\begin{tabular}{|c|c|}
\hline Coefficient & Value \\
\hline$c$ & 0.35 \\
\hline$e$ & 1.5 \\
\hline$g$ & 0.4 \\
\hline$K_{3}$ & 0.72 \\
\hline$\psi_{L}$ & $10^{\circ}$ \\
\hline
\end{tabular}

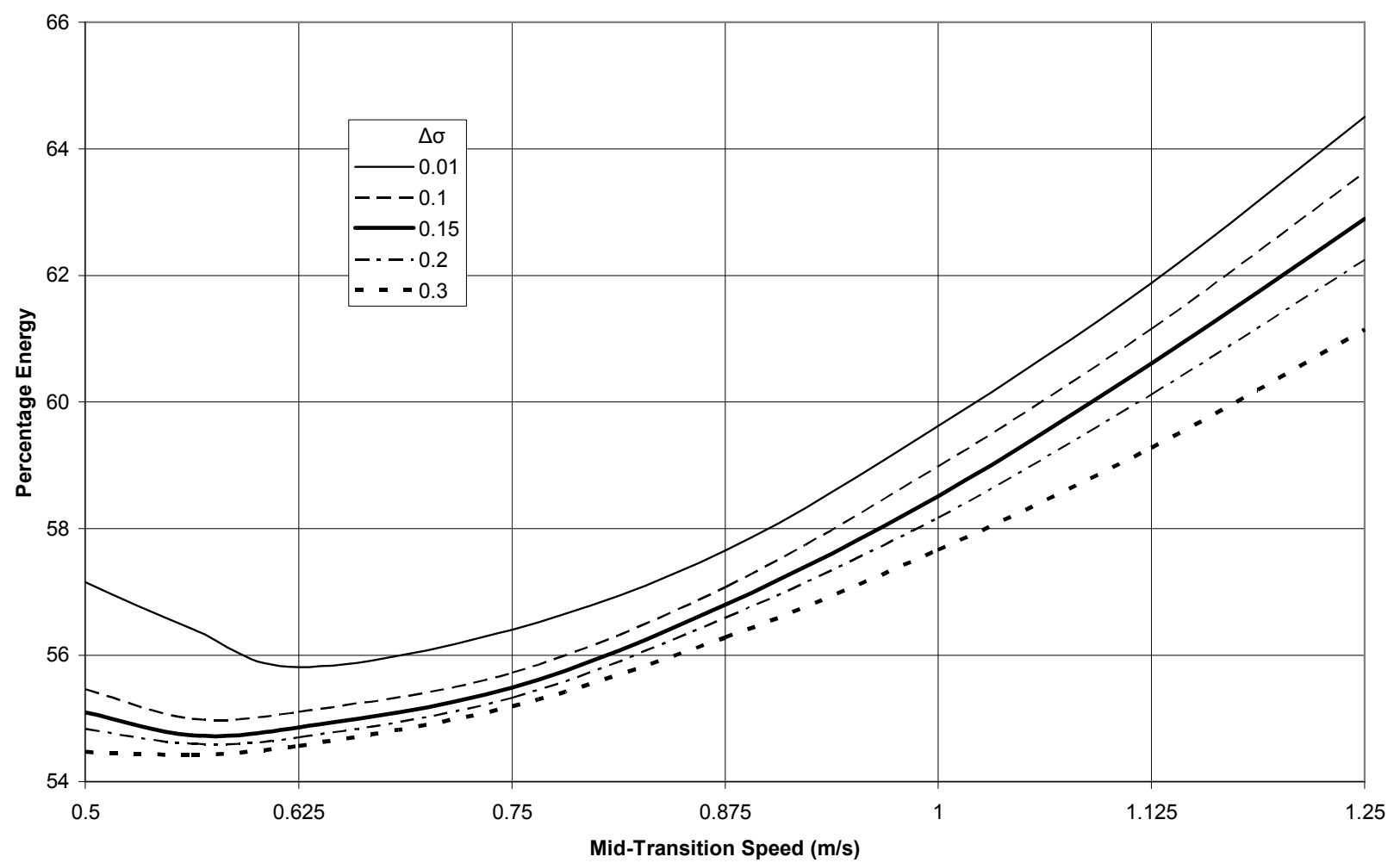

Figure 4 - Variation of required energy (vertical axis) with transition control

The measure of percentage energy presented in Figure 4 is taken as the energy used by the tunnel thrusters to return the vehicle to the original depth as a percentage of the energy required for the entire transition phase to be undertaken using tunnel thruster control alone. This assumes that the loss of performance of the stern mounted control surfaces, as the advance speed reduces, causes an inability to generate sufficient downwards force and hence leads to a change in depth.

The energy is calculated using the time history of the rotational speed of the tunnel thruster. This is converted into power, using a simple momentum theory based relationship, and hence the required energy can be calculated. Figure 4 shows the collated results of the simulations, for different $\bar{u}$ and $\Delta \sigma$.

Figure 4 shows that there is an optimum mid-transition speed and that the energy is relatively insensitive to the transition steepness. The increase in required energy at lower speeds is due to the increased depth change due to greater loss of control surface performance. The increase in required energy at higher speeds is due to the increased use, and decrease in performance, of the tunnel thruster at these speeds. It should be noted that the variations in required energy are small, that is, of the order of less than $10 \%$. The location of the minima on the curves indicates that going through the transition phase at lower speeds is favourable in terms of minimising energy usage however a consequence of this choice is a potentially significant depth change and potential loss of pitch control making the control system design an exercise in compromise.

\section{FUTURE WORK}

The future work consists of two main parts, firstly, the development and validation of the modelling framework proposed in this paper, and secondly, further investigations into the manoeuvring and control of positively buoyant 
survey-style AUVs over the entire speed range. The modelling framework and the justification behind the choices made are to be investigated through an experimental programme using a model scale torpedo-shaped AUV. This programme will focus on the three modes of operation considered in this paper. This is necessary to develop a robust framework for use on full-scale AUVs. This process will also allow the extension of the model to take account of the use of multiple thruster units. The investigations into manoeuvring and control will focus on the possible approaches to the transition phase and the incorporation of additional control devices on survey AUVs to provide low speed manoeuvring capabilities.

\section{CONCLUSIONS}

The proposed model, (9), and the insight gained from the literature review demonstrate how at the initial stages of design the optimum use of an additional control device in the form of a through-body tunnel thruster can be used to minimise demand on a limited energy supply.

This work also demonstrates the role of simplified models in the development of AUVs and how they can provide insight into vehicle operation. The case presented shows an example analysis of how a simulation can aid in control system design and mission planning by providing insight into the operation of a positively buoyant AUV undergoing transition from survey operation to low speed manoeuvring in terms of the energy used and the desired depth and pitch control.

\section{REFERENCES}

Bachmayer, R., L.L. Whitcomb and M.A. Grosenbaugh (2000). An accurate four-quadrant nonlinear dynamical model for marine thrusters: theory and experimental validation. $J-O E$, vol. 25, no. 1, pp 146-159.

Blanke, M., K.-P. Lindegaard and T.I. Fossen (2000). Dynamic model for thrust generation of marine propellers. In: IFAC 5th conference on maneuvering and control of marine craft. Aalborg, Denmark, 23-25 Aug. 2000. Elsevier, Kidlington, UK.

Breivik, M. and T.I. Fossen, (2006). A unified control concept for autonomous underwater vehicles. In: IEEE 2006 american control conference. Minneapolis, MN, United States, 14-16 Jun. 2006. IEEE, Piscataway, NJ, USA.

Brix, J.E. and O. Bussemaker (1973). Lateral thrusters with anti-suction tunnels. In: Ship \& Boat International $1 s t$ north american tug convention. Vancouver, Canada, Apr.-May 1973. Reed Industrial Press Ltd., London, UK.

Carlton, J.S. (2007). Marine propellers and propulsion. $2^{\text {nd }}$ ed. Oxford: Butterworth-Heinemann.

Chislett, M.S. and O. Björheden (1966). Influence of ship speed on the effectiveness of a lateral-thrust unit. Report Hy-8, Hydro-og Aerodynamisk Laboratorium, Lyngby, Denmark.

Cody, S.E. (1992). Experimental study of the response of small tunnel thrusters to triangular and square wave inputs. Masters Thesis, Naval Postgraduate School, Monterrey, CA, USA.
English, J.W. (1963). The design and performance of lateral thrust units for ships. Trans. RINA, vol. 105, no. 3, pp 251-279.

Fric, T.F. and A. Roshko (1994). Vortical structure in the wake of a transverse jet. JFM, vol. 279, pp 1-48.

Furlong, M. (2005). System identification of the hydrodynamic characteristics of underwater vehicles. Engineering Doctorate Thesis, University of Southampton.

Godhavn, J.-M., T.I. Fossen and S.P. Berge (1998). Nonlinear and adaptive backstepping designs for tracking control of ships. Internat. J. Adapt. Control Signal Process., vol. 12, no. 8, pp 649-670.

Gopalan, S., B.M. Abraham and J. Katz (2004). The structure of a jet in crossflow at low velocity ratios. Phys. Fluids, vol. 16, no. 6, pp 2067-2087.

Healey, A.J., S.M. Rock, S. Cody, D. Miles and J.P. Brown (1995). Toward an improved understanding of thruster dynamics for underwater vehicles. $J-O E$, vol. 20, no. 4, pp 354-361.

Karlikov, V.P. and G.I. Sholomovich (1998). Some features of body-flow interaction in the presence of transverse jets. Fluid Dynam., vol. 33, no. 3, pp 313-317.

Kim, J. and W.K. Chung (2006). Accurate and practical thruster modeling for underwater vehicles. Ocean Eng., vol. 33, pp 566-586.

Kimber, N.I. and W.B. Marshfield (1993). Design and testing of control surfaces for the autosub demonstrator test vehicle. DRA Haslar Technical Report (restricted access).

McLean, M.B. (1991). Dynamic performance of small diameter tunnel thrusters. Masters Thesis, Naval Postgraduate School, Monterrey, CA, USA.

Newman, P., R. Westwood and J. Westwood (2007). Market prospects for autonomous underwater vehicles. In: Reed Exhibitions The $9^{\text {th }}$ unmanned underwater vehicle showcase, Southampton, UK, 26-27 Sept. 2007. Reed Exhibitions Ltd, London

Nienhuis, U. (1987). Simulation of low frequency motions of dynamically positioned offshore structures. Trans. RINA, vol. 129, pp 127-145.

Nienhuis, U. (1992). Analysis of thruster effectivity for dynamic positioning and low speed manoeuvring. $\mathrm{PhD}$ Thesis, Delft University.

Saunders, A. \& M. Nahon (2002). The effect of forward vehicle velocity on through-body AUV tunnel thruster performance. In: MTS/IEEE Oceans 2002 - marine frontiers, Biloxi, MI, USA, 29-31 Oct. 2002. IEEE, Piscataway, NJ, USA.

Symons, P.J.R. and J.A. Sadden (1982). The design of the seabed operations vessel. Trans. RINA., vol. 124, pp 4161.

van Lammeran, W.P.A., J.D. van Manen and M.W.C. Oosterveld (1969). The wageningen B-screw series. Trans. SNAME, vol. 77, pp 269-317.

Yoerger, D.R., J.G. Cooke and J.-J.E. Slotine (1990). The influence of thruster dynamics on underwater vehicle behavior and their incorporation into control system design. $J-O E$, vol. 15, no. 3, pp 167-178. 